\title{
Systematic review of the influence of chemotherapy- associated liver injury on outcome after partial hepatectomy for colorectal liver metastases
}

Citation for published version (APA):

Zhao, J., van Mierlo, K. M. C., Gómez-Ramírez, J., Kim, H., Pilgrim, C. H. C., Pessaux, P., Rensen, S. S., van der Stok, E. P., Schaap, F. G., Soubrane, O., Takamoto, T., Viganò, L., Winkens, B., Dejong, C. H. C., Olde Damink, S. W. M., \& Chemotherapy-Associated Liver Injury (CALI) consortium (2017). Systematic review of the influence of chemotherapy-associated liver injury on outcome after partial hepatectomy for colorectal liver metastases. British Journal of Surgery, 104(8), 990-1002.

https://doi.org/10.1002/bjs.10572

Document status and date:

Published: 01/07/2017

DOI:

10.1002/bjs. 10572

Document Version:

Publisher's PDF, also known as Version of record

Document license:

Taverne

Please check the document version of this publication:

- A submitted manuscript is the version of the article upon submission and before peer-review. There can be important differences between the submitted version and the official published version of record.

People interested in the research are advised to contact the author for the final version of the publication, or visit the DOI to the publisher's website.

- The final author version and the galley proof are versions of the publication after peer review.

- The final published version features the final layout of the paper including the volume, issue and page numbers.

Link to publication

\footnotetext{
General rights rights.

- You may freely distribute the URL identifying the publication in the public portal. please follow below link for the End User Agreement:

www.umlib.nl/taverne-license

Take down policy

If you believe that this document breaches copyright please contact us at:

repository@maastrichtuniversity.nl

providing details and we will investigate your claim.
}

Copyright and moral rights for the publications made accessible in the public portal are retained by the authors and/or other copyright owners and it is a condition of accessing publications that users recognise and abide by the legal requirements associated with these

- Users may download and print one copy of any publication from the public portal for the purpose of private study or research.

- You may not further distribute the material or use it for any profit-making activity or commercial gain

If the publication is distributed under the terms of Article $25 \mathrm{fa}$ of the Dutch Copyright Act, indicated by the "Taverne" license above, 


\title{
Systematic review of the influence of chemotherapy-associated liver injury on outcome after partial hepatectomy for colorectal liver metastases
}

\author{
J. Zhao' ${ }^{1}$ (i) K. M. C. van Mierlo ${ }^{1}$, J. Gómez-Ramírez ${ }^{5}$, H. Kim ${ }^{6}$, C. H. C. Pilgrim ${ }^{7}$, P. Pessaux ${ }^{8}$, \\ S. S. Rensen ${ }^{1}$, E. P. van der Stok ${ }^{4}$, F. G. Schaap ${ }^{1}$, O. Soubrane ${ }^{9}$, T. Takamoto ${ }^{10}$, L. Viganò ${ }^{11}$, \\ B. Winkens ${ }^{2}$, C. H. C. Dejong1,3 and S. W. M. Olde Damink ${ }^{1,12}$, on behalf of the \\ Chemotherapy-Associated Liver Injury (CALI) consortium
}

${ }^{1}$ Department of Surgery, Maastricht University Medical Centre and NUTRIM School of Nutrition and Translational Research in Metabolism, ${ }^{2}$ Department of Methodology and Statistics, Maastricht University Medical Centre, and CAPHRI School for Public Health and Primary Care, and ${ }^{3}$ GROW School for Oncology and Developmental Biology, Maastricht University, Maastricht, and ${ }^{4}$ Department of Surgical Oncology, Erasmus MC Cancer Institute, Rotterdam, The Netherlands, ${ }^{5}$ Hepatopancreaticobiliary Surgery Unit, Department of Surgery, Hospital Universitario de la Princesa, Madrid, Spain, ${ }^{6}$ Department of Pathology, Seoul National University Bundang Hospital, Seoul National College of Medicine, Seongnam, Korea, ${ }^{7}$ Hepatopancreaticobiliary Service, Upper Gastrointestinal Surgery, The Alfred Hospital, and Division of Cancer Surgery, Peter MacCallum Cancer Centre, Department of Surgery, University of Melbourne, St Vincent's Hospital, Melbourne, Victoria, Australia, ${ }^{8}$ Digestive Surgery and Transplantation, Hôpital de Hautepierre, University Hospital of Strasbourg, Strasbourg, and ${ }^{9}$ Department of Hepatobiliary Surgery and Liver Transplant, Beaujon Hospital, Assistance Publique - Hôpitaux de Paris, Université Denis Diderot, Paris, France, ${ }^{10}$ Department of Hepatopancreaticobiliary Surgery, Japanese Red Cross Medical Centre, Tokyo, Japan, ${ }^{11}$ Division of Hepatobiliary and General Surgery, Humanitas Clinical and Research Centre, Humanitas University, Rozzano, Italy, and ${ }^{12}$ Department of Hepatopancreaticobiliary Surgery and Liver Transplantation, Institute for Liver and Digestive Health, Royal Free Hospital, University College London, London, UK

Correspondence to: Professor S. W. M. Olde Damink, Department of Surgery, Maastricht University Medical Centre and NUTRIM School of Nutrition and Translational Research in Metabolism, Maastricht University, Universiteitssingel 50, 6229 ER Maastricht, The Netherlands (e-mail: steven.oldedamink@maastrichtuniversity.nl; @ @UNFANGZHAO | @MaastrichtU | @maasuniversity | @MaastrichtUMC)

Background: The impact of chemotherapy-associated liver injury (CALI) on postoperative outcome in patients undergoing partial hepatectomy for colorectal liver metastases (CRLM) remains controversial. The objective of this study was to clarify the effect of CALI (sinusoidal dilatation (SD), steatosis and steatohepatitis) on postoperative morbidity and mortality by investigating a large data set from multiple international centres.

Methods: PubMed and Embase were searched for studies published between 1 January 2004 and 31 December 2013 with keywords 'chemotherapy', 'liver resection', 'outcome' and 'colorectal metastases' to identify potential collaborating centres. Univariable and multivariable analyses were performed using binary logistic regression models, with results presented as odds ratios (ORs) with 95 per cent confidence intervals.

Results: A consolidated database comprising 788 patients who underwent hepatectomy for CRLM in eight centres was obtained. In multivariable analyses, severe SD was associated with increased major morbidity (Dindo-Clavien grade III-V; OR 1.73, 95 per cent c.i. 1.02 to $2.95 ; P=0.043$ ). Severe steatosis was associated with decreased liver surgery-specific complications (OR 0.52, 95 per cent c.i. 0.27 to 1.00; $P=0.049)$, whereas steatohepatitis was linked to an increase in these complications (OR 2.08, 1.18 to 3.66; $P=0.012)$. Subgroup analysis showed that lobular inflammation was the sole component associated with increased overall morbidity (OR $2 \cdot 22,1.48$ to $3.34 ; P=0.001)$ and liver surgery-specific complications (OR 3.35, 2.11 to $5 \cdot 32 ; P<0.001)$. Finally, oxaliplatin treatment was linked to severe SD (OR 2.74, 1.67 to $4.49 ; P<0.001)$.

Conclusion: An increase in postoperative major morbidity and liver surgery-specific complications was observed after partial hepatectomy in patients with severe SD and steatohepatitis. Postoperative liver failure occurred more often in patients with severe SD. 
Presented to the International Liver Congress 2016, Barcelona, Spain, April 2016, and the 12th World Congress of the International Hepato-Pancreato-Biliary Association, São Paulo, Brazil, April 2016; published in abstract form as 7 Hepatol 2016; 64: S235 and HPB 2016; 18(Suppl 1): e58

Paper accepted 29 March 2017

Published online 25 May 2017 in Wiley Online Library (www.bjs.co.uk). DOI: 10.1002/bjs.10572

\section{Introduction}

Colorectal cancer is the third most common cancer worldwide, affecting over 1.3 million patients annually ${ }^{1}$. Approximately 50 per cent of these patients develop colorectal liver metastases $(\mathrm{CRLM})^{2,3}$. Although liver resection provides the best prospect of cure, only 10-30 per cent of patients with liver metastases are eligible for hepatic surgery ${ }^{3,4}$. For patients with tumours deemed unresectable, neoadjuvant systemic chemotherapy can prolong survival, and potentially allow future hepatic resection ${ }^{2,5}$.

For decades, 5-fluorouracil (5-FU) was the sole option for treating CRLM. This has changed markedly in the new millennium; with the approval of irinotecan, oxaliplatin and humanized monoclonal antibodies, approximately 15 per cent of patients with initially unresectable tumours became eligible for liver resection ${ }^{6,7}$. Unfortunately, administration of irinotecan- and/or oxaliplatin-based chemotherapeutic agents has been associated with a harmful side-effect in the form of liver injury ${ }^{8,9}$.

Chemotherapy-associated liver injury (CALI) is often reported in patients with CRLM, and appears to be regimen-specific. For instance, oxaliplatin treatment is associated with sinusoidal obstruction syndrome $(\mathrm{SOS})^{10}$, and linked to increased occurrence of nodular regenerative hyperplasia $(\mathrm{NRH})^{11}$. Co-administration of bevacizumab with oxaliplatin, however, has been reported to be associated with a decrease in both the incidence and severity of $\mathrm{SOS}$ and $\mathrm{NRH}^{10-12}$. Irinotecan-based regimens appear to be related to the development of steatohepatitis ${ }^{8,13,14}$. Importantly, because patients commonly receive several chemotherapeutic agents to offer optimal benefit in downsizing tumours, it is difficult to identify the specific agents responsible for injury to the hepatic parenchyma.

Certain studies $913,15-18$ have claimed a negative correlation between CALI and postoperative outcome (postoperative morbidity, mortality), but others ${ }^{19-23}$ could not reproduce this. Therefore, it remains unclear whether CALI influences postoperative morbidity and mortality. The aim of the present study was to explore whether sinusoidal dilatation (SD), steatosis and steatohepatitis are associated with increased morbidity and mortality rates after partial hepatectomy by performing a meta-analysis of individual participant data based on a systematic literature review. Additionally, factors associated with the occurrence of CALI were identified.

\section{Methods}

An extensive protocol, written before the start of this study, can be found in Appendix S1 (supporting information). PRISMA and MOOSE guidelines were followed when conducting and reporting this review ${ }^{24,25}$.

\section{Search strategy for identification of studies}

Systematic searches were performed in MEDLINE (PubMed) and Embase for studies published between 1 January 2004 and 31 December 2013 using a search matrix including the following four categories: liver resection, chemotherapy, tumour type and outcome. For the purpose of performing a more comprehensive search, the type of liver injury was not included in the search matrix. The full search strategy is shown in Table S1 (supporting information). The first publication date was fixed at 2004 because the widely used criteria for scoring SD, steatosis and steatohepatitis were developed in $2004^{26}$ and $2005^{27}$. No language filter was applied.

\section{Inclusion criteria}

Studies meeting the following criteria were considered eligible for inclusion: adult patients (aged over 18 years), who underwent liver resection for CRLM, with description of postoperative short-term overall morbidity, liver surgery-specific complications, postoperative liver failure or overall mortality (within 90 days or in-hospital) after liver resection, and with pathological assessment of non-tumorous liver specimens for SD, steatosis and/or steatohepatitis (Table $S 2$, supporting information). Studies with patients who received preoperative hepatic arterial infusion of chemotherapy were excluded, as were case reports, comments, those with published abstracts only, editorials and reviews.

\section{Study identification and data collection}

Duplicates were removed. Two authors independently screened all titles and abstracts and excluded those not pertinent to the review. Any discrepancy was resolved by consensus. The remaining articles were included for full-text revision and assessed independently for eligibility by the two authors. Reference lists of fully reviewed articles were checked manually for additional potential citations, along 
with exploration of a personal library that was established during previous research on this topic ${ }^{28,29}$.

\section{Definitions}

SD was graded according to Rubbia-Brandt and colleagues $^{26}$, with grade $2-3$ (severe SD) considered clinically relevant as it reflects rupture of sinusoidal wall integrity. SD is one of the most important histological features of SOS, and its severity is generally accepted to correspond to the severity of SOS. Steatosis and steatohepatitis were graded according to the system of Kleiner et al. ${ }^{27}$. Severe steatosis was defined as more than 33 per cent of parenchyma affected by steatosis $^{27}$. A non-alcoholic steatohepatitis activity score (NAS) of 4 or more was considered as steatohepatitis to provide a working cut-off value conforming with the literature ${ }^{30}$. CALI was defined as any occurrence of severe SD, severe steatosis or steatohepatitis. Comorbidity was defined as any disease affecting the patient apart from CRLM (diabetes mellitus, pulmonary, renal, cardiovascular and other diseases). Overall morbidity was defined as any complication occurring within 90 days after surgery or during the hospital stay, and graded according to the classification of Dindo and colleagues ${ }^{31}$. Major morbidity was defined as Dindo-Clavien grade III (requiring invasive intervention) or higher. The concept of a liver surgery-specific complication was analogous to the liver surgery-specific composite endpoint developed in 2011, and included one or more of the following events: ascites, postoperative liver failure, bile leakage, intra-abdominal abscess, intra-abdominal haemorrhage and operative mortality ${ }^{32}$. Postoperative liver failure was defined as the concurrent presence of a prothrombin time below 50 per cent and a serum bilirubin level greater than $50 \mu \mathrm{mol} / \mathrm{l}$ (the $50-50$ criteria) on or after postoperative day $5^{33}$. Postoperative mortality included death from any cause within 90 days after surgery or during the hospital admission. Major hepatectomy was defined as resection of three or more Couinaud liver segments ${ }^{34}$.

\section{Risk-of-bias assessment}

The risk of bias of the final included studies was assessed independently by two blinded researchers using the Quality in Prognosis Studies (QUIPS) tool ${ }^{35,36}$. Discrepancies were discussed by the two authors and consensus was reached. The QUIPS tool includes six bias domains: participation, attrition, prognostic factor measurement, confounding measurement and account, outcome measurement, and analysis and reporting. The results of assessment of each of the six domains were taken together to calculate an overall low, moderate or high risk of bias, as follows: overall low risk of bias, two or fewer domains rated as moderate risk and the remaining domains as low risk; overall moderate risk, at least three domains rated as moderate risk and the remaining domains as low risk; and high overall risk, one or more domains rated as high risk, independent of the rating of the remaining domains.

\section{Data handling and statistical analysis}

Corresponding authors from studies that fulfilled the inclusion criteria were contacted by e-mail for collaboration and sharing of coded data (by numbering) for the published cohort. Each author was asked to sign a specific data transfer agreement form, which assured careful handling of the data. Coded data were arranged in a preconstructed Excel $^{\mathrm{TM}}$ file (Microsoft, Redmond, Washington, USA) and subsequently imported into SPSS ${ }^{\circledR}$ for Windows ${ }^{\circledR}$ version 20.0 (IBM, Armonk, New York, USA).

Patient characteristics were compared using the Student's $t$ test for numerical variables. For categorical variables, the Pearson $\chi^{2}$ test with continuity correction was applied, or Fisher's exact test when any of the expected values was smaller than five. The influence of preoperative chemotherapeutic agents on liver injury, and subsequently the effect of liver injury on short-term postoperative outcome, were analysed by applying one-step binary logistic regression models. Individual participant data from all studies were pooled and modelled simultaneously. This approach was considered more optimal because each study showed relatively few events per outcome and small sample sizes, and the one-step approach for pooled data allowed the exact binomial distribution to be used and did not require continuity corrections when there were no events $^{37,38}$. For missing values, multiple imputations were performed, assuming that data were missing at random. The number of imputations was determined by the maximum percentage of missing data in the data set. In this study, 30 imputations were performed, as the maximum amount of missing data was 26 per cent (minor/major morbidity). Variables in multiple imputations are listed in TableS3 (supporting information). A complete-case sensitivity analysis was also conducted. Clustering of patients from different studies was integrated as a separate co-variable ('database source') and included in binary logistic regression models in every analysis ${ }^{38}$. A single variable together with database source created the univariable model. All variables with $P \leq 0.200$ in univariable analysis were included in the multivariable analysis. In addition, database source and variables known to be related to the outcome (either well described in literature or based on 


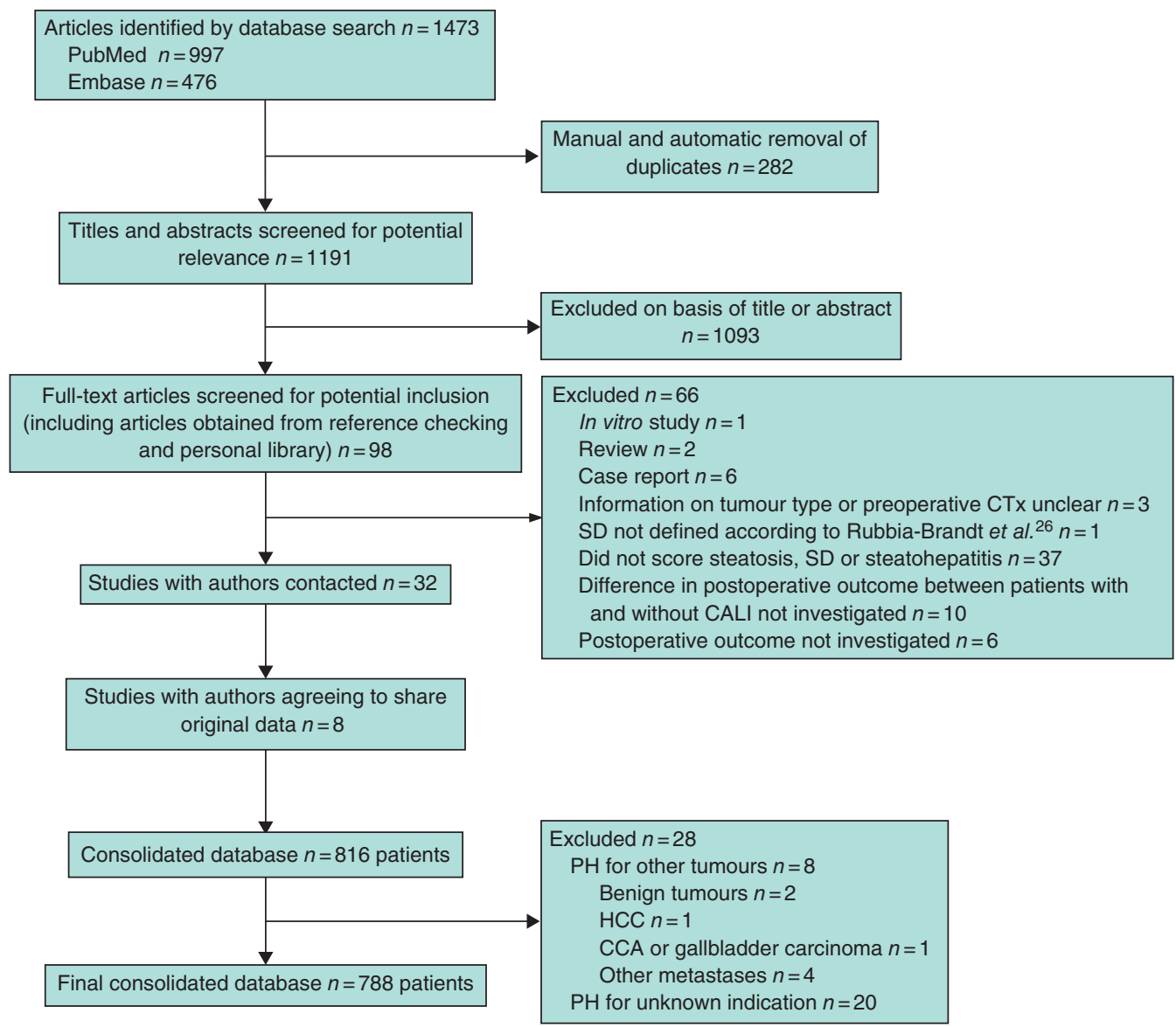

Fig. 1 Flow chart showing selection of articles for review. CTx, chemotherapy; SD, sinusoidal dilatation; CALI, chemotherapy-associated liver injury; PH, partial hepatectomy; HCC, hepatocellular carcinoma; CCA, cholangiocarcinoma

careful discussion and consensus between the authors) were forced into the multivariable model.

A subgroup analysis including only patients who received oxaliplatin-based treatment was performed to investigate the impact of bevacizumab on the occurrence of severe SD. At a later stage of the study, data to investigate the influence of separate NAS subcategories (steatosis, lobular inflammation and hepatocellular ballooning) on postoperative outcome were requested. A subgroup analysis including cohorts with available data on NAS subcategories was undertaken. The methods of analysis of these data were identical to those described above. Unadjusted and adjusted odds ratios (ORs) with 95 per cent confidence intervals were calculated. $P \leq 0.050$ was considered significant for all analyses.

\section{Results}

The search strategy resulted in 1191 unique hits (Fig. 1). In total, 1093 articles were excluded on the basis of title or abstract, and the remaining 98 articles were subjected to full-text evaluation. Thirty-two studies met the inclusion criteria and the 30 corresponding authors were contacted by e-mail. Nineteen responded, of whom eight agreed to share their raw data. Other authors could not be contacted (Fig. S1, supporting information). Potential publication bias was excluded by testing for asymmetry in a funnel plot. The eight included studies ${ }^{16,17,39-44}$ were assessed for risk of bias using the QUIPS tool. Overall ratings are shown in Table 1. Six articles showed an overall low risk of bias and one study each had a moderate or high risk. Detailed scoring can be found in TableS4 (supporting information). Because the individual participant data from all studies were pooled and modelled simultaneously, an overall rating was also calculated without taking the study confounding and statistical analysis domains into account; all studies then showed a low risk of bias based on the remaining four domains.

Characteristics of the included studies are summarized in Table 1. The consolidated cohort consisted of 816 patients 
Table 1 Characteristics of studies included in final database

\begin{tabular}{|c|c|c|c|c|c|c|}
\hline Reference & Country & $\begin{array}{l}\text { Study } \\
\text { type }\end{array}$ & Population* & $\begin{array}{l}\text { QUIPS } \\
\text { score }\end{array}$ & Comparisons & Key findings \\
\hline $\begin{array}{l}\text { Gómez-Ramírez } \\
\text { et al. }{ }^{39}\end{array}$ & Spain & PCS & $45(46)$ & Low & $\begin{array}{l}\text { Neoadjuvant CTx versus } \\
\text { no CTx }\end{array}$ & $\begin{array}{l}\text { Patients treated with oxaliplatin } \\
\text { had a higher incidence of SOS, } \\
\text { an increase in liver } \\
\text { complications and longer mean } \\
\text { hospital stay }\end{array}$ \\
\hline Nam et al. ${ }^{40}$ & Korea & RCS & 89 & Low & $\begin{array}{c}\text { Oxaliplatin CTx versus } \\
\text { non-oxaliplatin CTx }\end{array}$ & $\begin{array}{l}\text { Sinusoidal injury is frequently seen } \\
\text { in oxaliplatin-treated livers and } \\
\text { should be documented in } \\
\text { surgical pathology practice } \\
\text { when extensive }\end{array}$ \\
\hline Pessaux et al. ${ }^{41}$ & France & $\mathrm{RCC}$ & $52(36)$ & High & $\begin{array}{l}\text { Neoadjuvant CTx versus } \\
\text { CTx }+ \\
\text { cetuximab/bevacizumab }\end{array}$ & $\begin{array}{l}\text { The addition of bevacizumab or } \\
\text { cetuximab to the neoadjuvant } \\
\text { CTx does not increase } \\
\text { morbidity rates after } \\
\text { hepatectomy for CRLM }\end{array}$ \\
\hline Pilgrim et al. ${ }^{16}$ & Australia & RCS & 232 & Low & $\begin{array}{l}\text { Severe versus non-severe } \\
\text { CALI }\end{array}$ & $\begin{array}{l}\text { Severe steatosis was associated } \\
\text { with increased postoperative } \\
\text { morbidity, whereas severe } \\
\text { steatohepatitis and sinusoidal } \\
\text { injury were not }\end{array}$ \\
\hline Soubrane et al. ${ }^{42}$ & France & RCS & $78(105)$ & Low & $\begin{array}{l}\text { Severe versus non-severe } \\
\text { SD }\end{array}$ & $\begin{array}{l}\text { SOS } 2-3 \text { was associated with } \\
\text { postoperative hepatic } \\
\text { dysfunction and ascites after } \\
\text { major liver resection }\end{array}$ \\
\hline Takamoto et al. ${ }^{43}$ & Japan & RCS & 55 (104) & Moderate & $\begin{array}{l}\text { Liver injury versus no liver } \\
\text { injury }\end{array}$ & $\begin{array}{l}\text { Hepatic functional reserve, } \\
\text { represented by the ICG-R15 } \\
\text { value, improves during the } \\
\text { period after cessation of } \\
\text { chemotherapy }\end{array}$ \\
\hline van der Pool et al. ${ }^{44}$ & The Netherlands & $\mathrm{RCS}$ & 104 & Low & $\begin{array}{c}\text { Neoadjuvant CTx versus } \\
\text { CTx + bevacizumab }\end{array}$ & $\begin{array}{l}\text { Bevacizumab added to } \\
\text { oxaliplatin-based CTx may } \\
\text { protect against moderate SD } \\
\text { without significantly influencing } \\
\text { morbidity }\end{array}$ \\
\hline Viganò et al. ${ }^{17}$ & Italy & PCS & 100 & Low & $\begin{array}{l}\text { Severe versus non-severe } \\
\text { CALI }\end{array}$ & $\begin{array}{l}\text { Liver biopsy cannot be considered } \\
\text { a reliable tool in assessing CALI } \\
\text { except for steatosis. Proportion } \\
\text { of liver dysfunction was higher } \\
\text { among patients with CALI }\end{array}$ \\
\hline
\end{tabular}

*Values are number of patients in cohort reported in published article, with number in shared database in parentheses. QUIPS, Quality in Prognosis Studies; PCS, prospective cohort study; CTx, chemotherapy; SOS, sinusoidal obstruction syndrome; RCS, retrospective cohort study; RCC, retrospective case-control study; CRLM, colorectal liver metastases; CALI, chemotherapy-associated liver injury; SD, sinusoidal dilatation; ICG-R15, indocyanine green retention rate at $15 \mathrm{~min}$.

(Fig. 1). Twenty-eight patients were excluded because they underwent surgery for indications other than CRLM, leaving 788 patients for analysis. Patient characteristics, surgical details and postoperative outcomes of the consolidated cohort are shown in Table 2. There were 453 men $(57.5$ per cent) and 335 women $(42.5$ per cent), with a median age of 61 (range 25-86) years. SD was severe in 183 patients ( 24.1 per cent), steatosis was severe in 117 (15.6 per cent) and steatohepatitis in $100(14.5$ per cent). Of the 525 patients who received 5-FU, 396 (75.4 per cent) had simultaneous treatment with oxaliplatin and 135 (25.7 per cent) with irinotecan. Of the 136 patients who received capecitabine, 119 (87.5 per cent) had simultaneous treatment with oxaliplatin. Cetuximab and bevacizumab were most frequently administered together with oxaliplatin. All 61 patients who received cetuximab, and 138 of 164 ( 84.1 per cent) who received bevacizumab, were co-treated with oxaliplatin. Of 635 patients with NAS subcategory data, 96 (15.1 per cent) had a NAS of 4 or more. Only three patients ( 3 per cent) with a NAS of 4 or higher did not show lobular inflammation. The relationship between NAS and lobular inflammation is summarized in Table S5 (supporting information).

Sensitivity analysis showed similar results for completecase analysis and that with multiple imputation (detailed information available on request). The influence of severe $\mathrm{SD}$, severe steatosis, steatohepatitis and other potential factors related to short-term overall morbidity, liver 
Table 2 Patient, surgical and postoperative characteristics

\begin{tabular}{|c|c|c|c|c|c|c|c|c|c|c|}
\hline & \multirow[b]{2}{*}{$n$} & \multicolumn{3}{|c|}{ Sinusoidal dilatation $(n=760)$} & \multicolumn{3}{|c|}{ Steatosis $(n=752)$} & \multicolumn{3}{|c|}{ Steatohepatitis $(n=690)$} \\
\hline & & $\begin{array}{c}\text { Non-severe } \\
(n=577)\end{array}$ & $\begin{array}{c}\text { Severe } \\
(n=183)\end{array}$ & $P \dagger$ & $\begin{array}{c}\text { Non-severe } \\
(n=635)\end{array}$ & $\begin{array}{c}\text { Severe } \\
(n=117)\end{array}$ & $P$ & $\begin{array}{l}\text { NAS }<4 \\
(n=590)\end{array}$ & $\begin{array}{l}N A S \geq 4 \\
(n=100)\end{array}$ & $P \dagger$ \\
\hline Age (years) ${ }^{\star}$ & 788 & $61(11)$ & $60(10)$ & $0.576 \ddagger$ & $61(11)$ & $60(9)$ & $0.527 \ddagger$ & $61(11)$ & $61(10)$ & 0.784 \\
\hline Sex & & & & 0.836 & & & 1.000 & & & 0.784 \\
\hline M & 453 & $335(58.1)$ & $104(56 \cdot 8)$ & & $366(57.6)$ & $68(58.1)$ & & $336(56.9)$ & $59(59.0)$ & \\
\hline $\mathrm{F}$ & 335 & $242(41.9)$ & 79 (43.2) & & $269(42.4)$ & 49 (41.9) & & $254(43 \cdot 1)$ & $41(41.0)$ & \\
\hline BMI $\left(\mathrm{kg} / \mathrm{m}^{2}\right)^{\star}$ & 706 & $25 \cdot 3(4 \cdot 4)$ & $24.4(3.9)$ & $0.001 \ddagger$ & $24 \cdot 7(4 \cdot 1)$ & $27 \cdot 6(4 \cdot 1)$ & $<0.001 \ddagger$ & $24 \cdot 9(4 \cdot 2)$ & $26 \cdot 7(3.9)$ & $<0.001 \neq$ \\
\hline Co-morbidity & & & & 0.259 & & & 0.012 & & & 0.158 \\
\hline No & 363 & $247(49 \cdot 3)$ & $93(54.7)$ & & $290(52 \cdot 2)$ & $41(38.3)$ & & $271(51.4)$ & $31(42)$ & \\
\hline Yes & 336 & $254(50 \cdot 7)$ & $77(45 \cdot 3)$ & & $266(47 \cdot 8)$ & $66(61.7)$ & & $256(48 \cdot 6)$ & $43(58)$ & \\
\hline Preoperative chemotherapy & & & & 0.001 & & & 0.032 & & & 0.198 \\
\hline No & 127 & $110(19 \cdot 3)$ & $15(8.4)$ & & $114(18.2)$ & $11(9 \cdot 6)$ & & $112(19 \cdot 3)$ & $13(13)$ & \\
\hline Yes & 649 & $459(80 \cdot 7)$ & $164(91.6)$ & & $511(81.8)$ & $104(90 \cdot 4)$ & & $468(80 \cdot 7)$ & $85(87)$ & \\
\hline $5-\mathrm{FU}$ & & & & 0.006 & & & 0.463 & & & 0.396 \\
\hline No & 245 & $201(35 \cdot 6)$ & $43(24.2)$ & & $210(34.0)$ & $34(29.8)$ & & $213(37 \cdot 0)$ & $31(32)$ & \\
\hline Yes & 525 & $363(64.4)$ & $135(75 \cdot 8)$ & & $408(66.0)$ & $80(70.2)$ & & $362(63.0)$ & $66(68)$ & \\
\hline Capecitabine & & & & 0.173 & & & 0.158 & & & 0.061 \\
\hline No & 634 & $454(80.5)$ & $152(85.4)$ & & $511(82.4)$ & $87(76 \cdot 3)$ & & $466(81.0)$ & $70(72)$ & \\
\hline Yes & 136 & $110(19.5)$ & $26(14.6)$ & & $109(17 \cdot 6)$ & $27(23.7)$ & & $109(19.0)$ & $27(28)$ & \\
\hline Irinotecan & & & & 0.033 & & & 0.033 & & & 1.000 \\
\hline No & 627 & $445(78.9)$ & $154(86.5)$ & & $508(81.9)$ & $83(72 \cdot 8)$ & & $453(78 \cdot 8)$ & $77(79)$ & \\
\hline Yes & 143 & $119(21 \cdot 1)$ & $24(13.5)$ & & $112(18.1)$ & $31(27 \cdot 2)$ & & $122(21 \cdot 2)$ & $20(21)$ & \\
\hline Bevacizumab & & & & 0.072 & & & 0.143 & & & 0.101 \\
\hline No & 605 & $430(76 \cdot 4)$ & $148(83 \cdot 1)$ & & $487(78 \cdot 7)$ & $82(71.9)$ & & $444(77.4)$ & $67(69)$ & \\
\hline Yes & 164 & $133(23.6)$ & $30(16.9)$ & & $132(21 \cdot 3)$ & $32(28 \cdot 1)$ & & $130(22.6)$ & $30(31)$ & \\
\hline Cetuximab & & & & 0.198 & & & 0.716 & & & 0.462 \\
\hline No & 708 & $522(92 \cdot 7)$ & $159(89.3)$ & & $566(91.4)$ & $106(93.0)$ & & $522(90.9)$ & $91(94)$ & \\
\hline Yes & 61 & $41(7 \cdot 3)$ & $19(10 \cdot 7)$ & & $53(8.6)$ & $8(7.0)$ & & $52(9.1)$ & $6(6)$ & \\
\hline Oxaliplatin & & & & $<0.001$ & & & 0.716 & & & 0.295 \\
\hline No & 266 & $228(40.5)$ & $37(20 \cdot 8)$ & & $226(36 \cdot 5)$ & $39(34.2)$ & & $231(40 \cdot 2)$ & $33(34)$ & \\
\hline Yes & 503 & $335(59.5)$ & $141(79 \cdot 2)$ & & $393(63.5)$ & $75(65 \cdot 8)$ & & $343(59 \cdot 8)$ & $64(66)$ & \\
\hline Resection type & & & & $<0.001$ & & & 0.921 & & & 0.713 \\
\hline Minor ( $<3$ segments) & 398 & $330(57 \cdot 2)$ & $67(36.8)$ & & $335(52 \cdot 8)$ & $63(53.8)$ & & $339(57 \cdot 6)$ & $55(55.0)$ & \\
\hline Major ( $\geq 3$ segments) & 389 & $247(42 \cdot 8)$ & $115(63.2)$ & & $299(47 \cdot 2)$ & $54(46 \cdot 2)$ & & $250(42.4)$ & $45(45 \cdot 0)$ & \\
\hline PVE & & & & 0.001 & & & 0.046 & & & 0.512 \\
\hline No & 623 & $461(92.0)$ & $140(82.4)$ & & $498(89.6)$ & $103(96 \cdot 3)$ & & 491 (93.2) & $71(96)$ & \\
\hline Yes & 76 & $40(8.0)$ & $30(17 \cdot 6)$ & & $58(10.4)$ & $4(3.7)$ & & $36(6 \cdot 8)$ & $3(4)$ & \\
\hline Pringle manoeuvre & & & & 0.018 & & & 0.974 & & & 1.000 \\
\hline No & 446 & $347(64.5)$ & $93(54.1)$ & & $366(62 \cdot 1)$ & $71(62 \cdot 8)$ & & $349(63.9)$ & $61(64)$ & \\
\hline Yes & 291 & $191(35.5)$ & $79(45.9)$ & & $223(37.9)$ & $42(37.2)$ & & $197(36 \cdot 1)$ & $34(36)$ & \\
\hline Transfusion of packed RBCs & & & & 0.004 & & & 0.871 & & & 0.382 \\
\hline No & 646 & $492(86 \cdot 2)$ & $139(76 \cdot 8)$ & & $529(84.4)$ & $100(85.5)$ & & $508(86 \cdot 8)$ & $83(83.0)$ & \\
\hline Yes & 134 & $79(13.8)$ & $42(23 \cdot 2)$ & & $98(15 \cdot 6)$ & $17(14.5)$ & & $77(13.2)$ & $17(17.0)$ & \\
\hline Postoperative short-term outcomes & & & & & & & & & & \\
\hline Length of hospital stay (days) ${ }^{\star}$ & 768 & $14(13)$ & $15(11)$ & $0 \cdot 240 \ddagger$ & $15(11)$ & $14(17)$ & $0.660 \%$ & $14(12)$ & $15(14)$ & $0 \cdot 266 \ddagger$ \\
\hline Overall morbidity & & & & 0.009 & & & 0.935 & & & 0.024 \\
\hline No & 443 & $339(59 \cdot 3)$ & $87(47 \cdot 8)$ & & $360(57 \cdot 3)$ & $66(56.4)$ & & $355(60 \cdot 6)$ & $48(48.0)$ & \\
\hline Yes & 338 & $233(40 \cdot 7)$ & $95(52.2)$ & & $268(42 \cdot 7)$ & $51(43.6)$ & & $231(39.4)$ & $52(52.0)$ & \\
\hline None/minor morbidity (DC 0-II) & 491 & $362(88.5)$ & $106(73 \cdot 1)$ & $<0.001$ & $397(85.6)$ & $69(85)$ & 1.000 & $367(89.5)$ & $62(81)$ & 0.041 \\
\hline Major morbidity (DC III-V) & 89 & $47(11.5)$ & $39(26.9)$ & & $67(14.4)$ & $12(15)$ & & $43(10.5)$ & $15(19)$ & \\
\hline Liver surgery-specific complication & & & & $<0.001$ & & & 0.031 & & & 0.247 \\
\hline No & 598 & $455(79 \cdot 8)$ & $121(66.9)$ & & $475(75.9)$ & $100(85.5)$ & & $470(80.6)$ & $75(75.0)$ & \\
\hline Yes & 180 & $115(20.2)$ & $60(33.1)$ & & $151(24.1)$ & $17(14.5)$ & & $113(19.4)$ & $25(25.0)$ & \\
\hline Mortality & & & & $0.200 \S$ & & & $1.000 \S$ & & & $0.422 \S$ \\
\hline No & 780 & $573(99 \cdot 3)$ & $179(97.8)$ & & $628(98.9)$ & $116(99.1)$ & & $586(99 \cdot 3)$ & $98(98.0)$ & \\
\hline Yes & 8 & $4(0.7)$ & $4(2 \cdot 2)$ & & $7(1 \cdot 1)$ & $1(0.9)$ & & $4(0.7)$ & $2(2 \cdot 0)$ & \\
\hline Postoperative liver failure & & & & $<0.001$ & & & $0.302 \S$ & & & $1.000 \$$ \\
\hline No & 755 & $563(98.6)$ & $166(91 \cdot 7)$ & & $608(97.0)$ & $116(99.1)$ & & $578(99.0)$ & $99(99.0)$ & \\
\hline Yes & 24 & $8(1.4)$ & $15(8 \cdot 3)$ & & $19(3.0)$ & $1(0.9)$ & & $6(1.0)$ & $1(1.0)$ & \\
\hline
\end{tabular}

Value in parentheses are percentages unless indicated otherwise; *values are mean(s.d.). Data represent original data without multiple imputations; owing to missing values, some numbers do not add up to the total number of patients. NAS, non-alcoholic fatty liver disease activity score; 5-FU, 5-fluorouracil; PVE, portal vein embolization; RBC, red blood cell; DC, Dindo-Clavien grade. $\dagger$ Pearson $\chi^{2}$ test with continuity correction, except $\ddagger$ Student's $t$ test and $\S$ Fisher's exact test. 

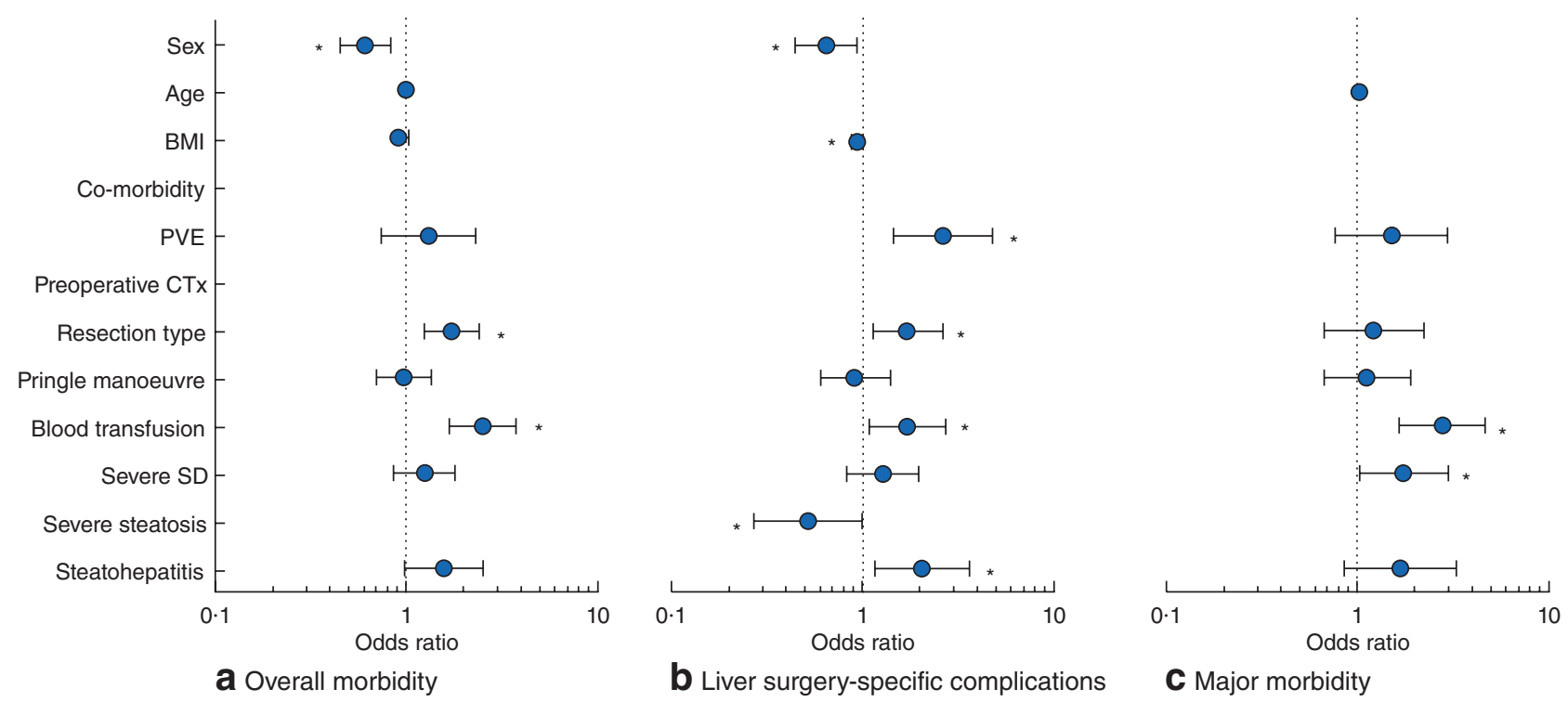

Fig. 2 Multivariable analysis of influence of liver injury on short-term postoperative outcomes: a overall morbidity, b liver surgery-specific complications and $\mathbf{c}$ major morbidity. Odds ratios are shown with 95 per cent confidence intervals. PVE, portal vein embolization; CTx, chemotherapy; $\mathrm{SD}$, sinusoidal dilatation. ${ }^{*} P<0 \cdot 050$

Table 3 Univariable analyses of factors influencing short-term postoperative outcome

\begin{tabular}{|c|c|c|c|c|}
\hline & \multicolumn{2}{|c|}{ Postoperative liver failure $(n=24)$} & \multicolumn{2}{|c|}{ Mortality $(n=8)$} \\
\hline & Odds ratio & $P$ & Odds ratio & $P$ \\
\hline $\operatorname{Sex}(F)$ & $0.65(0.25,1.68)$ & 0.376 & $0.50(0.10,2.25)$ & 0.408 \\
\hline Age (years) & $1.00(0.96,1.04)$ & 0.937 & $1.03(0.96,1.05)$ & 0.402 \\
\hline $\mathrm{BMI}\left(\mathrm{kg} / \mathrm{m}^{2}\right)$ & $0.90(0.80,1.01)$ & 0.070 & $0.96(0.80,1.14)$ & 0.630 \\
\hline Co-morbidity & $1.28(0.53,3.13)$ & 0.583 & $7 \cdot 84(0.92,66 \cdot 65)$ & 0.059 \\
\hline PVE & $2.62(0.95,7.26)$ & 0.064 & $1.09(0.13,9.09)$ & 0.938 \\
\hline Preoperative chemotherapy & $2 \cdot 41(0.30,19 \cdot 32)$ & 0.409 & $1.51(0.52,4.42)$ & 0.702 \\
\hline Resection type (major) & $-^{*}$ & $-^{\star}$ & $6.56(0.77,55 \cdot 90)$ & 0.085 \\
\hline Pringle manoeuvre & $1.45(0.57,3.72)$ & 0.437 & $1.91(0.43,8.54)$ & 0.399 \\
\hline Blood transfusion & $4.47(1.69,11.82)$ & 0.003 & $14.00(2.74,71.51)$ & 0.002 \\
\hline Severe SD & $3.06(1.18,7.92)$ & 0.021 & $2.79(0.68,11.44)$ & 0.155 \\
\hline Severe steatosis & $0.47(0.06,3.77)$ & 0.473 & $0.86(0.10,7.09)$ & 0.887 \\
\hline Steatohepatitis & $1.90(0.31,22 \cdot 83)$ & 0.492 & $2.71(0.57,12.87)$ & 0.210 \\
\hline
\end{tabular}

Values in parentheses are 95 per cent confidence intervals. PVE, portal vein embolization; SD, sinusoidal dilatation. Multiple imputations were used for univariable analysis. *All patients with liver failure underwent major hepatectomy and none had minor hepatectomy $(P<0 \cdot 001$, Fisher's exact test).

surgery-specific complications and major morbidity is shown in Fig. 2 and TableS6 (supporting information). Severe SD was significantly associated with increased major morbidity only (OR $1.73,95$ per cent c.i. 1.02 to $2.95 ; P=0.043)$. Severe steatosis was not significantly associated with the occurrence of postoperative overall or major morbidity, but was related to a decreased occurrence of liver surgery-specific complications (OR $0 \cdot 52,0 \cdot 27$ to $1 \cdot 00 ; P=0 \cdot 049)$. In contrast, patients with steatohepatitis showed a significantly increased rate of postoperative liver surgery-specific complications (OR $2.08,1.18$ to $3.66 ; P=0.012)$, and a trend towards increased overall morbidity (OR 1.58, 0.99 to 2.52; $P=0.057)$.

Table 3 summarizes the effects of several factors associated with postoperative liver failure and mortality. Because of the small number of events, multivariable logistic regression was not performed. Postoperative liver failure occurred in 24 of 779 patients (3.1 per cent) in the study cohort (Table 2), seven women and $17 \mathrm{men}$, with a median age of 61 (range 48-75) years. All patients with liver failure had undergone major hepatectomy. The only factors strongly associated with increased liver failure in univariable analysis were severe SD (OR 3.06, 1.18 to 


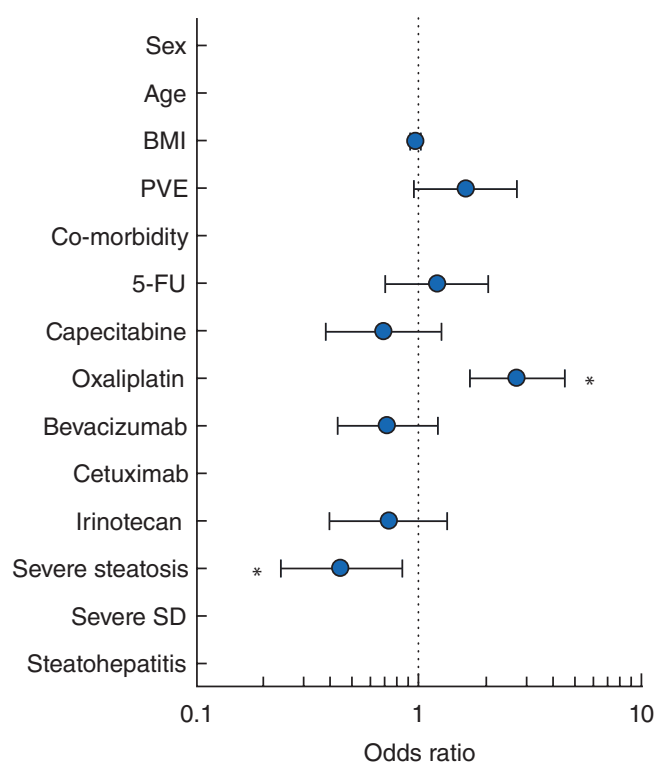

a Severe sinusoidal dilatation

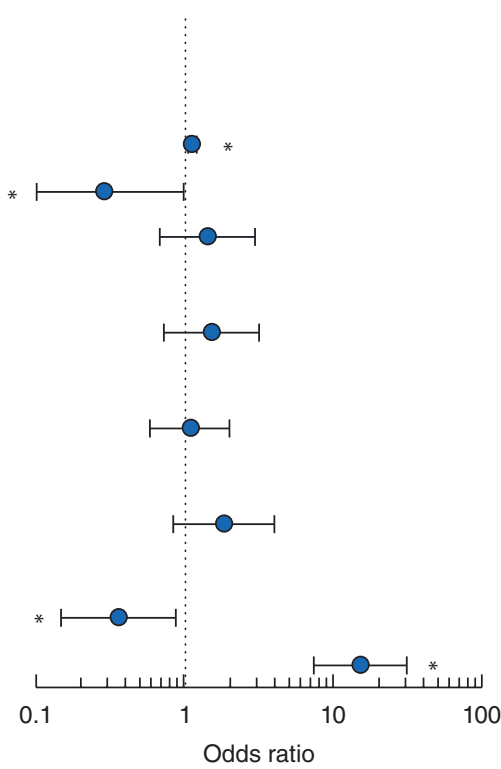

b Severe steatosis

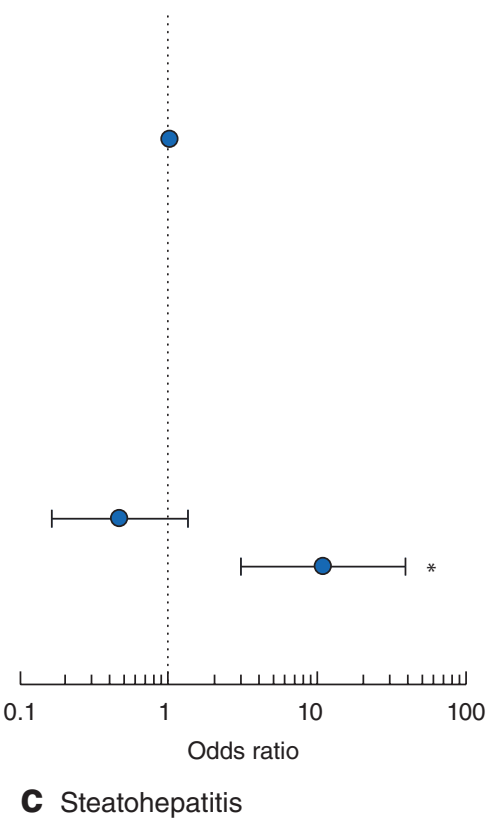

Fig. 3 Multivariable analysis of influence of risk factors on liver injury: a severe sinusoidal dilatation, $\mathbf{b}$ severe steatosis and $\mathbf{c}$ steatohepatitis. Odds ratios are shown with 95 per cent confidence intervals. PVE, portal vein embolization; 5-FU, 5-fluorouracil. ${ }^{*} P<0.050$

7.92; $P=0.021)$ and perioperative blood transfusion (OR 4.47, 1.69 to $11.82 ; P=0.003)$.

In total, eight patients ( 1.0 per cent $)$ died in the perioperative period after liver resection, seven within 90 days and one at 101 days during the hospital stay. The six men and two women had a median age of 64 (range 48-75) years. Perioperative blood transfusion (OR 14.00, 2.74 to $71.51 ; P=0.002)$ was the sole factor related to increased mortality in univariable analysis. A trend was found for major liver resection (OR 6.56, 0.77 to $55.90 ; P=0.085$ ) and preoperative co-morbidity (OR 7.84, 0.92 to 66.65 ; $P=0.059)$ to be associated with increased postoperative mortality. Severe SD (OR 2.79, 0.68 to $11.44 ; P=0.155)$, severe steatosis (OR $0.86,0.10$ to $7.09 ; P=0.887$ ) and steatohepatitis (OR 2.71, 0.57 to $12.87 ; P=0.210$ ) were not related to postoperative mortality.

Because steatohepatitis, but not severe steatosis, negatively affected postoperative short-term outcomes, lobular inflammation and hepatocellular ballooning were considered key factors for poor outcome. A subgroup analysis supported this hypothesis. In multivariable analyses, severe (grade 2-3) lobular inflammation was associated with increased overall postoperative morbidity (OR $2.22,1.48$ to $3.34 ; P=0.001)$ and liver surgery-specific morbidity (OR $3 \cdot 35,2 \cdot 11$ to $5 \cdot 32 ; P<0.001$ ), but not major morbidity (OR $1.63,0.85$ to $3.10 ; P=0 \cdot 138$ ). In contrast, neither severe steatosis (over 33 per cent) nor the presence of hepatocellular ballooning (grade 1-2) was associated with an increased complication rate in all multivariable analyses.

Associations between several preoperative variables and the occurrence of severe SD, severe steatosis and steatohepatitis are summarized in Fig. 3 and Table S7 (supporting information). Oxaliplatin (OR 2.74, 1.67 to 4.49; $P<0.001)$ was related to increased occurrence of severe $\mathrm{SD}$ in multivariable analysis, whereas the addition of bevacizumab was associated with a twofold decrease in severe $\mathrm{SD}$ in patients who received oxaliplatin (OR $0.50,0.30$ to $0.82 ; P=0.006)$ when the analysis was adjusted solely by database source. Patients with severe steatosis showed a decreased incidence of severe SD (OR 0.44, 0.24 to 0.83 ; $P=0 \cdot 011$ ) and vice versa (OR $0 \cdot 36,0.15$ to $0.88 ; P=0.025$ ). $\mathrm{BMI}$ was related to an increase in severe steatosis (OR $1.15,1.08$ to $1.21 ; P<0.001)$, whereas the incidence of severe steatosis was decreased in patients with portal vein embolization (PVE) (OR 0.29, 0.08 to $1.00 ; P=0.050$ ). Only severe steatosis was significantly associated with an increased occurrence of steatohepatitis (OR 15.09, 6.25 to 36.45; $P<0 \cdot 001)$.

\section{Discussion}

In this study, an increase in postoperative major morbidity and liver surgery-specific complications after partial 
hepatectomy was observed in patients with severe SD and steatohepatitis respectively, whereas severe steatosis was associated with a decreased occurrence of liver surgeryspecific complications. Postoperative liver failure occurred more often in patients with severe SD. With respect to steatohepatitis, lobular inflammation, but not severe steatosis or hepatocellular ballooning, was strongly linked to increased postoperative morbidity. Oxaliplatin-based chemotherapy was independently associated with an increase in the occurrence of severe SD, whereas a decrease was seen with the addition of bevacizumab. An inverse relationship was found between severe SD and severe steatosis.

Mechanisms underlying the negative influence of severe SD on major morbidity are unknown, although preoperative hepatic dysfunction, impairment of liver regeneration, Kupffer cell dysfunction, enhanced blood loss due to haemorrhagic pools, fragility of the liver, and increased hepatocellular necrosis as seen in human and animal models, can all be reasons for poor liver function and an increased complication rate after severe $\mathrm{SD}^{45,46}$. However, as the $P$ value was of borderline significance, the effect of severe $\mathrm{SD}$ on major morbidity must be interpreted with caution.

The progression of steatohepatitis is estimated by the so-called NAS, which is composed of scores for steatosis, lobular inflammation and hepatocellular ballooning ${ }^{27}$. Multivariable subgroup analyses examining the influence of these separate NAS subcategories on postoperative outcome showed a detrimental influence of lobular inflammation on all outcomes, whereas severe steatosis and hepatocellular ballooning had no effect. The mechanism behind steatohepatitis being a determinant of postoperative short-term outcomes is uncertain. Electron microscopy revealed that mitochondrial structural defects in hepatocytes correlate with steatohepatitis, but not with steatosis ${ }^{47}$. Moreover, the ability of the liver to recover from adenosine $5^{\prime}$-triphosphate depletion was severely impaired ${ }^{48}$, liver regeneration was diminished ${ }^{49}$, and humoral and cellular immune responses to enhanced oxidative stress were found in patients with steatohepatitis. These factors may account for postoperative complications.

Severe steatosis did not significantly influence short-term overall morbidity, major morbidity or mortality after partial hepatectomy in this study, which is in line with previous reports $^{50,51}$, but in contrast to findings in other studies ${ }^{52,53}$. Patients with severe steatosis even showed a decreased occurrence of liver surgery-specific complications. This might be explained by surgeons being more careful during surgery when observing a severely steatotic ('yellow') liver. However, with a $P$ value of 0.049 , this evidence needs to be validated by future research.
Severe SD was more common in patients who received oxaliplatin than among those who did not (29.6 versus 14.0 per cent). This is in line with previous studies ${ }^{10,17}$ that showed a similarly high rate of severe SD after oxaliplatin-based regimens. The addition of bevacizumab, an angiogenesis inhibitor, to oxaliplatin-based chemotherapy has been associated with a decreased incidence of $\mathrm{SD}^{44}$. Indeed, when the analysis was restricted to the population that received oxaliplatin-based treatment, bevacizumab was associated with a greatly decreased occurrence of severe SD. Although mechanisms underpinning those observations remain unclear, activation of vascular endothelial growth factor and coagulation pathways in oxaliplatin-related SD might be involved ${ }^{54}$.

Importantly, severe steatosis was linked to a decreased occurrence of severe SD and vice versa, raising the possibility that these events are mutually exclusive. Several phenomena could underlie this observation. First, mechanical pressure exerted by fat-laden, swollen hepatocytes may distort the hepatic microvasculature. In mice with severe steatosis, a decrease in sinusoidal perfusion, loss of fenestrae and narrowing of the sinusoidal lumen were observed $^{55}$. SD may therefore not develop owing to spatial constraints. Conversely, atrophied hepatocellular plates in severe SD may render these hepatocytes incapable of fatty acid uptake. Alternatively, histological assessment may be more challenging in a liver affected by both severe SD and steatosis, increasing the likelihood of misclassification of either one of these injury types. However, several pathologists with hepatobiliary expertise independently considered this probability very small. Although the reduction in SD in patients with severe steatosis is interesting, central pathology review is needed to verify this finding.

Apart from oxaliplatin, preoperative PVE was marginally associated with an increased occurrence of severe SD. A previous study ${ }^{56}$ has already acknowledged a possible influence of PVE on the development of vascular injury, probably by induction of ischaemia. The present authors hypothesize that the hepatic artery buffer response after PVE might play an even more profound role, as shown by the induction of microvascular remodelling and SD in the embolized lobe after portal branch ligation in a rodent model ${ }^{57}$. However, the morphology of the non-embolized remnant lobe may differ from that of the resected lobe, and liver histology and function may therefore not be affected after resection ${ }^{58}$.

In the present study, severe SD was less common in patients receiving additional bevacizumab than among those receiving oxaliplatin alone. Severe SD was shown to be associated with an increased major complication rate and vice versa. Although the effect of co-administration of 
oxaliplatin with bevacizumab on surgical outcome could not be investigated directly here, adding bevacizumab might provide an advantageous effect on postoperative outcome in patients treated with oxaliplatin.

Parenchymal damage due to chemotherapy can be diagnosed before surgery by radiological and biochemical means, as reviewed recently in detail ${ }^{59}$. Despite (experimental) research focusing on CALI, little evidence is available for its treatment in the human setting ${ }^{59}$. When liver injury is confirmed before operation, surgeons are thus advised to adapt surgical management to prevent complications. In the present study, the transfusion of packed red blood cells was associated with an increased postoperative complication rate, in line with previous literature ${ }^{60}$. Central venous pressure should be low during surgery to prevent excessive blood loss. Moreover, major hepatectomy was confirmed to be associated with an increased postoperative complication rate, which encourages minimization of the resection volume. Performing wedge resections instead of hemihepatectomy, and the use of radiofrequency ablation might be beneficial when feasible.

This study demonstrated both the effect of chemotherapy on liver injury and the subsequent effect of liver injury on short-term postoperative outcome in a large multicentre patient cohort. However, some limitations of the study should be discussed. First, NRH has recently caused concern because of its relationship to increased postoperative morbidity ${ }^{11}$. Although analysis of the effect of NRH on postoperative outcome would have been of interest, this was not possible owing to lack of available data. This may be because the data used in this review were from studies published before or around 2013, when NRH had not yet gained the attention it deserves. Inclusion of NRH is recommended when exploring the relationship between CALI and postoperative outcomes in future studies. Next, data on the interval between cessation of chemotherapy and partial hepatectomy, as well as the number of cycles administered, were not available for every study cohort. Therefore, the influence of these factors on the occurrence of CALI and short-term complications could not be evaluated. In addition, central review of all histopathology slides would have strengthened the paper substantially. Unfortunately, this was not feasible for logistical reasons. It must be highlighted, nonetheless, that all sections were reviewed by local pathologists with hepatobiliary expertise, and assessed according to uniform, well established and globally accepted scoring systems for SD, steatosis and steatohepatitis. Finally, as no RCTs exist on the topic, mainly retrospective cohort studies were included in this review. Despite this limitation, all included studies had a low risk of bias and nearly all original data could be retained, making this the most comprehensive multicentre data set currently available.

Considering the negative relationship between CALI and postoperative morbidity, it is advised to adapt surgical management when CALI is diagnosed. Moreover, with decreased chemotherapy responsiveness ${ }^{28,61}$, shortened overall survival ${ }^{62}$ and increasing doubts about the usefulness of neoadjuvant chemotherapy in certain patient groups $^{63}$, it could even be speculated that some patients would benefit from immediate resection instead of neoadjuvant chemotherapy. Prospective registration such as the ALPPS (Associated Liver Partition and Portal vein Ligation for Staged hepatectomy) Registry ${ }^{64}$ provides a way to obtain a higher level of evidence on this topic.

\section{Collaborators}

Other members of the CALI consortium are: I. García Sanz, E. Martín Pérez (Hepatopancreaticobiliary Surgery Unit, Department of Surgery, Hospital Universitario de la Princesa, Madrid, Spain); J. Y. Cho, Y. R. Choi (Department of Surgery, Seoul National University Bundang Hospital, Seoul National College of Medicine, Seongnam, Korea); W. Phillips, M. Michael (Division of Cancer Surgery, Peter MacCallum Cancer Centre, Department of Surgery, University of Melbourne, St Vincent's Hospital, Melbourne, Victoria, Australia); F. Panaro (Digestive Surgery and Transplantation, Hôpital de Hautepierre, University Hospital of Strasbourg, University of Strasbourg, Strasbourg, France); M.-P. Chenard (Department of Pathology, Hôpital de Hautepierre, University Hospital of Strasbourg, University of Strasbourg, Strasbourg, France); C. Verhoef, D. J. Grünhagen (Department of Surgical Oncology, Erasmus MC Cancer Institute, Rotterdam, The Netherlands); J. Vara (Digestive Tumours Unit, Institut Bergonié, Bordeaux, France); O. Scatton (Department of Digestive and Hepatobiliary Surgery, La Pitié Hospital, Université Pierre et Marie Curie, Paris, France); T. Hashimoto, M. Makuuchi (Department of Hepatopancreaticobiliary Surgery, Japanese Red Cross Medical Centre, Tokyo, Japan); G. De Rosa, N. Ravarino (Department of Pathology, Mauriziano Umberto I Hospital, Turin, Italy).

\section{Acknowledgements}

J.Z. and K.M.C.v.M. contributed equally to this study. The authors thank H. Nguyen for thorough reading and commenting on the manuscript. J.Z. is supported by the China Scholarship Council (201207040056).

Disclosure: The authors declare no conflict of interest.

\section{References}

1 Ferlay J, Soerjomataram I, Dikshit R, Eser S, Mathers C, Rebelo $\mathrm{M}$ et al. Cancer incidence and mortality worldwide: sources, methods and major patterns in GLOBOCAN 2012. Int 7 Cancer 2015; 136: E359-E386.

2 Leonard GD, Brenner B, Kemeny NE. Neoadjuvant chemotherapy before liver resection for patients with unresectable liver metastases from colorectal carcinoma. 7 Clin Oncol 2005; 23: 2038-2048. 
3 Kanas GP, Taylor A, Primrose JN, Langeberg WJ, Kelsh MA, Mowat FS et al. Survival after liver resection in metastatic colorectal cancer: review and meta-analysis of prognostic factors. Clin Epidemiol 2012; 4: 283-301.

4 Scheele J. Hepatectomy for liver metastases. Br 7 Surg 1993; 80: 274-276.

5 Tanaka K, Adam R, Shimada H, Azoulay D, Lévi F, Bismuth $H$. Role of neoadjuvant chemotherapy in the treatment of multiple colorectal metastases to the liver. Br 7 Surg 2003; 90: $963-969$.

6 Adam R, Wicherts DA, de Haas RJ, Ciacio O, Lévi F, Paule $\mathrm{B}$ et al. Patients with initially unresectable colorectal liver metastases: is there a possibility of cure? 7 Clin Oncol 2009; 27: 1829-1835.

7 Nordlinger B, Van Cutsem E, Rougier P, Köhne CH, Ychou M, Sobrero A et al.; European Colorectal Metastases Treatment Group. Does chemotherapy prior to liver resection increase the potential for cure in patients with metastatic colorectal cancer? A report from the European Colorectal Metastases Treatment Group. Eur 7 Cancer 2007; 43: 2037-2045.

8 Robinson SM, Wilson CH, Burt AD, Manas DM, White SA. Chemotherapy-associated liver injury in patients with colorectal liver metastases: a systematic review and meta-analysis. Ann Surg Oncol 2012; 19: 4287-4299.

9 Zorzi D, Laurent A, Pawlik TM, Lauwers GY, Vauthey JN, Abdalla EK. Chemotherapy-associated hepatotoxicity and surgery for colorectal liver metastases. Br 7 Surg 2007; 94 : $274-286$.

10 Rubbia-Brandt L, Lauwers GY, Wang H, Majno PE, Tanabe K, Zhu AX etal. Sinusoidal obstruction syndrome and nodular regenerative hyperplasia are frequent oxaliplatin-associated liver lesions and partially prevented by bevacizumab in patients with hepatic colorectal metastasis. Histopathology 2010; 56: 430-439.

11 Viganò L, Rubbia-Brandt L, De Rosa G, Majno P, Langella $\mathrm{S}$, Toso C et al. Nodular regenerative hyperplasia in patients undergoing liver resection for colorectal metastases after chemotherapy: risk factors, preoperative assessment and clinical impact. Ann Surg Oncol 2015; 22: 4149-4157.

12 Ribero D, Wang H, Donadon M, Zorzi D, Thomas MB, Eng C et al. Bevacizumab improves pathologic response and protects against hepatic injury in patients treated with oxaliplatin-based chemotherapy for colorectal liver metastases. Cancer 2007; 110: 2761-2767.

13 Vauthey JN, Pawlik TM, Ribero D, Wu TT, Zorzi D, Hoff $\mathrm{PM}$ et al. Chemotherapy regimen predicts steatohepatitis and an increase in 90-day mortality after surgery for hepatic colorectal metastases. 7 Clin Oncol 2006; 24: 2065-2072.

14 Khan AZ, Morris-Stiff G, Makuuchi M. Patterns of chemotherapy-induced hepatic injury and their implications for patients undergoing liver resection for colorectal liver metastases. 7 Hepatobiliary Pancreat Surg 2009; 16: 137-144.

15 Nakano H, Oussoultzoglou E, Rosso E, Casnedi S, Chenard-Neu MP, Dufour P et al. Sinusoidal injury increases morbidity after major hepatectomy in patients with colorectal liver metastases receiving preoperative chemotherapy. Ann Surg 2008; 247: 118-124.

16 Pilgrim CH, Satgunaseelan L, Pham A, Murray W, Link E, Smith $\mathrm{M}$ et al. Correlations between histopathological diagnosis of chemotherapy-induced hepatic injury, clinical features, and perioperative morbidity. $H P B$ 2012; 14 : $333-340$.

17 Viganò L, Ravarino N, Ferrero A, Motta M, Torchio B, Capussotti L. Prospective evaluation of accuracy of liver biopsy findings in the identification of chemotherapyassociated liver injuries. Arch Surg 2012; 147: 1085-1091.

18 Aloia T, Sebagh M, Plasse M, Karam V, Lévi F, Giacchetti S et al. Liver histology and surgical outcomes after preoperative chemotherapy with fluorouracil plus oxaliplatin in colorectal cancer liver metastases. 7 Clin Oncol 2006; 24: 4983-4990.

19 Komori H, Beppu T, Baba Y, Horino K, Imsung C, Masuda $\mathrm{T}$ et al. Histological liver injury and surgical outcome after FOLFOX followed by a hepatectomy for colorectal liver metastases in Japanese patients. Int 7 Clin Oncol 2010; 15: $263-270$.

20 Pawlik TM, Olino K, Gleisner AL, Torbenson M, Schulick R, Choti MA. Preoperative chemotherapy for colorectal liver metastases: impact on hepatic histology and postoperative outcome. 7 Gastrointest Surg 2007; 11: $860-868$.

21 Sahajpal A, Vollmer CM, Dixon E, Chan EK, Wei A, Cattral MS et al. Chemotherapy for colorectal cancer prior to liver resection for colorectal cancer hepatic metastases does not adversely affect peri-operative outcomes. 7 Surg Oncol 2007; 95: 22-27.

22 Makowiec F, Möhrle S, Neeff H, Drognitz O, Illerhaus G, Opitz OG et al. Chemotherapy, liver injury, and postoperative complications in colorectal liver metastases. f Gastrointest Surg 2011; 15: 153-164.

23 Kandutsch S, Klinger M, Hacker S, Wrba F, Gruenberger B, Gruenberger T. Patterns of hepatotoxicity after chemotherapy for colorectal cancer liver metastases. Eur 7 Surg Oncol 2008; 34: 1231-1236.

24 Moher D, Liberati A, Tetzlaff J, Altman DG; PRISMA Group. Preferred reporting items for systematic reviews and meta-analyses: the PRISMA statement. Ann Intern Med 2009; 151: 264-269, W264.

25 Stroup DF, Berlin JA, Morton SC, Olkin I, Williamson GD, Rennie D etal. Meta-analysis of observational studies in epidemiology: a proposal for reporting. Meta-analysis Of Observational Studies in Epidemiology (MOOSE) group. 7AMA 2000; 283: 2008-2012.

26 Rubbia-Brandt L, Audard V, Sartoretti P, Roth AD, Brezault C, Le Charpentier M et al. Severe hepatic sinusoidal obstruction associated with oxaliplatin-based chemotherapy in patients with metastatic colorectal cancer. Ann Oncol 2004; 15: 460-466.

27 Kleiner DE, Brunt EM, Van Natta M, Behling C, Contos MJ, Cummings OW etal. Design and validation of a 
histological scoring system for nonalcoholic fatty liver disease. Hepatology 2005; 41: 1313-1321.

28 Vreuls CP, Van Den Broek MA, Winstanley A, Koek GH, Wisse E, Dejong CH etal. Hepatic sinusoidal obstruction syndrome (SOS) reduces the effect of oxaliplatin in colorectal liver metastases. Histopathology 2012; 61: 314-318.

29 van Mierlo KMC, Zhao J, Kleijnen J, Rensen SS, Schaap FG, Dejong CHC et al. The influence of chemotherapyassociated sinusoidal dilatation on short-term outcome after partial hepatectomy for colorectal liver metastases: a systematic review with meta-analysis. Surg Oncol 2016; 25: 298-307.

30 Brunt EM, Kleiner DE, Wilson LA, Belt P, Neuschwander-Tetri BA; NASH Clinical Research Network (CRN). Nonalcoholic fatty liver disease (NAFLD) activity score and the histopathologic diagnosis in NAFLD: distinct clinicopathologic meanings. Hepatology 2011; 53: 810-820.

31 Dindo D, Demartines N, Clavien PA. Classification of surgical complications: a new proposal with evaluation in a cohort of 6336 patients and results of a survey. Ann Surg 2004; 240: 205-213.

32 van den Broek MA, van Dam RM, van Breukelen GJ, Bemelmans MH, Oussoultzoglou E, Pessaux P et al. Development of a composite endpoint for randomized controlled trials in liver surgery. Br 7 Surg 2011; 98: $1138-1145$.

33 Balzan S, Belghiti J, Farges O, Ogata S, Sauvanet A, Delefosse D et al. The '50-50 criteria' on postoperative day 5: an accurate predictor of liver failure and death after hepatectomy. Ann Surg 2005; 242: 824-828.

34 Couinaud C. Liver anatomy: portal (and suprahepatic) or biliary segmentation. Dig Surg 1999; 16: 459-467.

35 Hayden JA, van der Windt DA, Cartwright JL, Côté P, Bombardier C. Assessing bias in studies of prognostic factors. Ann Intern Med 2013; 158: 280-286.

36 Hayden JA, Côté P, Bombardier C. Evaluation of the quality of prognosis studies in systematic reviews. Ann Intern Med 2006; 144: 427-437.

37 Debray TP, Moons KG, Abo-Zaid GM, Koffijberg H, Riley RD. Individual participant data meta-analysis for a binary outcome: one-stage or two-stage? PLoS One 2013; 8: e60650.

38 Abo-Zaid G, Guo B, Deeks JJ, Debray TP, Steyerberg EW, Moons KG et al. Individual participant data meta-analyses should not ignore clustering. 7 Clin Epidemiol 2013; 66: $865-873 . e 864$.

39 Gómez-Ramírez J, Martín-Pérez E, Amat CG, Sanz IG, Bermejo E, Rodríguez A et al. Influence of pre-surgical chemotherapy on liver parenchyma and post-surgical outcome of patients subjected to hepatectomy due to colorectal carcinoma metastases. Cir Esp 2010; 88: 404-412.

40 Nam SJ, Cho JY, Lee HS, Choe G, Jang JJ, Yoon YS et al. Chemotherapy-associated hepatopathy in Korean colorectal cancer liver metastasis patients: oxaliplatin-based chemotherapy and sinusoidal injury. Korean 7 Pathol 2012; 46: $22-29$
41 Pessaux P, Marzano E, Casnedi S, Bachellier P, Jaeck D, Chenard MP. Histological and immediate postoperative outcome after preoperative cetuximab: case-matched control study. World 7 Surg 2010; 34: 2765-2772.

42 Soubrane O, Brouquet A, Zalinski S, Terris B, Brézault C, Mallet $\mathrm{V}$ et al. Predicting high grade lesions of sinusoidal obstruction syndrome related to oxaliplatin-based chemotherapy for colorectal liver metastases: correlation with post-hepatectomy outcome. Ann Surg 2010; 251: 454-460.

43 Takamoto T, Hashimoto T, Sano K, Maruyama Y, Inoue K, Ogata $\mathrm{S}$ et al. Recovery of liver function after the cessation of preoperative chemotherapy for colorectal liver metastasis. Ann Surg Oncol 2010; 17: 2747-2755.

44 van der Pool AE, Marsman HA, Verheij J, Ten Kate FJ, Eggermont AM, Ijzermans JN et al. Effect of bevacizumab added preoperatively to oxaliplatin on liver injury and complications after resection of colorectal liver metastases. 7 Surg Oncol 2012; 106: 892-897.

45 Schiffer E, Frossard JL, Rubbia-Brandt L, Mentha G, Pastor $\mathrm{CM}$. Hepatic regeneration is decreased in a rat model of sinusoidal obstruction syndrome. 7 Surg Oncol 2009; 99: 439-446.

46 Hubert C, Dahrenmoller C, Marique L, Jabbour N, Gianello P, Leclercq I. Hepatic regeneration in a rat model is impaired by chemotherapy agents used in metastatic colorectal cancer. Eur 7 Surg Oncol 2015; 41: 1471-1478.

47 Sanyal AJ, Campbell-Sargent C, Mirshahi F, Rizzo WB, Contos MJ, Sterling RK et al. Nonalcoholic steatohepatitis: association of insulin resistance and mitochondrial abnormalities. Gastroenterology 2001; 120: 1183-1192.

48 Cortez-Pinto H, Chatham J, Chacko VP, Arnold C, Rashid A, Diehl AM. Alterations in liver ATP homeostasis in human nonalcoholic steatohepatitis: a pilot study. $7 A M A$ 1999; 282: 1659-1664.

49 Yang SQ, Lin HZ, Mandal AK, Huang J, Diehl AM. Disrupted signaling and inhibited regeneration in obese mice with fatty livers: implications for nonalcoholic fatty liver disease pathophysiology. Hepatology 2001; 34: 694-706.

50 Wiggans MG, Lordan JT, Shahtahmassebi G, Aroori S, Bowles MJ, Stell DA. The interaction between diabetes, body mass index, hepatic steatosis, and risk of liver resection: insulin dependent diabetes is the greatest risk for major complications. HPB Surg 2014; 2014: 586159.

51 Reddy SK, Marsh JW, Varley PR, Mock BK, Chopra KB, Geller DA et al. Underlying steatohepatitis, but not simple hepatic steatosis, increases morbidity after liver resection: a case-control study. Hepatology 2012; 56: 2221-2230.

52 McCormack L, Petrowsky H, Jochum W, Furrer K, Clavien PA. Hepatic steatosis is a risk factor for postoperative complications after major hepatectomy: a matched case-control study. Ann Surg 2007; 245: 923-930.

53 Kooby DA, Fong Y, Suriawinata A, Gonen M, Allen PJ, Klimstra DS et al. Impact of steatosis on perioperative 
outcome following hepatic resection. 7 Gastrointest Surg 2003; 7: 1034-1044.

54 Rubbia-Brandt L, Tauzin S, Brezault C, Delucinge-Vivier C, Descombes P, Dousset B etal. Gene expression profiling provides insights into pathways of oxaliplatin-related sinusoidal obstruction syndrome in humans. Mol Cancer Ther 2011; 10: 687-696.

55 Farrell GC, Teoh NC, McCuskey RS. Hepatic microcirculation in fatty liver disease. Anat Rec (Hoboken) 2008; 291: 684-692.

56 Brouquet A, Benoist S, Julie C, Penna C, Beauchet A, Rougier $\mathrm{P}$ et al. Risk factors for chemotherapy-associated liver injuries: a multivariate analysis of a group of 146 patients with colorectal metastases. Surgery 2009; 145 : $362-371$.

57 Kollmar O, Corsten M, Scheuer C, Vollmar B, Schilling MK, Menger MD. Portal branch ligation induces a hepatic arterial buffer response, microvascular remodeling, normoxygenation, and cell proliferation in portal blood-deprived liver tissue. Am 7 Physiol Gastrointest Liver Physiol 2007; 292: 1534-1542.

58 Matsuo K, Murakami T, Kawaguchi D, Hiroshima Y, Koda $\mathrm{K}$, Yamazaki K et al. Histologic features after surgery associating liver partition and portal vein ligation for staged hepatectomy versus those after hepatectomy with portal vein embolization. Surgery 2016; 159: 1289-1298.

59 van Mierlo KM, Schaap FG, Dejong CH, Olde Damink SW. Liver resection for cancer: new developments in prediction, prevention and management of postresectional liver failure. 7 Hepatol 2016; 65: 1217-1231.

60 Farid SG, White A, Khan N, Toogood GJ, Prasad KR, Lodge JP. Clinical outcomes of left hepatic trisectionectomy for hepatobiliary malignancy. Br 7 Surg 2016; 103: 249-256.

61 Viganò L, Capussotti L, De Rosa G, De Saussure WO, Mentha G, Rubbia-Brandt L. Liver resection for colorectal metastases after chemotherapy: impact of chemotherapy-related liver injuries, pathological tumor response, and micrometastases on long-term survival. Ann Surg 2013; 258: 731-742.

62 Tamandl D, Klinger M, Eipeldauer S, Herberger B, Kaczirek K, Gruenberger B et al. Sinusoidal obstruction syndrome impairs long-term outcome of colorectal liver metastases treated with resection after neoadjuvant chemotherapy. Ann Surg Oncol 2011; 18: 421-430.

63 Khoo E, O’Neill S, Brown E, Wigmore SJ, Harrison EM. Systematic review of systemic adjuvant, neoadjuvant and perioperative chemotherapy for resectable colorectal-liver metastases. HPB 2016; 18: 485-493.

64 Schadde E, Ardiles V, Robles-Campos R, Malago M, Machado M, Hernandez-Alejandro R et al.; ALPPS Registry Group. Early survival and safety of ALPPS: first report of the International ALPPS Registry. Ann Surg 2014; 260: $829-836$.

\section{Supporting information}

Additional supporting information may be found in the online version of this article:

Appendix S1 Study protocol (Word document)

Table S1 Search strategy (Word document)

Table S2 Inclusion criteria (Word document)

Table S3 Variables in multiple imputations (Word document)

Table S4 Quality in Prognostic Studies detailed risk-of-bias assessment of individual included studies (Word document)

Table S5 Relationship between non-alcoholic fatty liver disease activity score and lobular inflammation (Word document)

Table S6 Influence of liver injury on short-term postoperative outcomes (Word document)

Table S7 Factors related to liver injury (Word document)

Fig. S1 Flow chart summarizing final inclusion process (Word document) 